\title{
Modelos da distribuição temporal de chuvas intensas em Piracicaba, SP
}

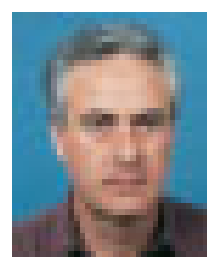

Décio E. Cruciani ${ }^{1}$, Ronalton E. Machado ${ }^{2}$ \& Paulo C. Sentelhas ${ }^{3}$

\author{
1 ESALQ/USP. Av. Pádua Dias, 11, CP 9, CEP 13418-900, Piracicaba, SP. E-mail: cruciani@carpa.ciagri.usp.br (Foto) \\ 2 ESALQ/USP, Pós-graduando. E-mail: remachad@carpa.ciagri.usp.br \\ ${ }^{3}$ ESALQ/USP. E-mail: pcsentel@carpa.ciagri.usp.br
}

Protocolo $009-9 / 1 / 2001$

\begin{abstract}
Resumo: O estudo da variação temporal de chuvas intensas é de grande importância na hidrologia, para a análise e previsão de eventos extremos, necessárias em projetos de controle de engenharia. Com esse objetivo, foram analisados dados de pluviogramas da cidade de Piracicaba, SP, do período de 1966 a 2000, para se determinar a distribuição temporal de chuvas intensas de 60 e de 120 min de duração. As chuvas de 60 min foram subdivididas em três intervalos iguais e sucessivos de 20 min cada um, enquanto as chuvas de $120 \mathrm{~min}$ foram subdivididas em quatro intervalos iguais e sucessivos de 30 min cada um. O modelo de distribuição da precipitação que predominou para as chuvas de 60 e $120 \mathrm{~min}$, foi do tipo exponencial negativo, com 85,7 e $50,7 \%$ dos casos, respectivamente. Para as chuvas de $60 \mathrm{~min}$, com altura pluviométrica média de $20,7 \mathrm{~mm}$, a distribuição foi de $72,3,21,4$ e $6,2 \%$ do total precipitado, respectivamente, nos três intervalos sucessivos de $20 \mathrm{~min}$. Para as chuvas de $120 \mathrm{~min}$, com altura pluviométrica média de $33,3 \mathrm{~mm}$, o resultado foi de $60,1,25,2,11,1$ e $3,6 \%$, respectivamente, nos quatro intervalos sucessivos de $30 \mathrm{~min}$. O modelo de distribuição temporal dessas chuvas não foi modificado pelo total precipitado nem pela sua duração, nos intervalos em questão.
\end{abstract}

Palavras-chave: chuvas intensas, distribuição temporal, modelos, probabilidade

\section{Time distribution models of intense rainfall in Piracicaba, SP, Brazil}

\begin{abstract}
Time distribution models of intense and short rains are very important in hydrology and for extreme predictions in engineering projects. With this purpose, rain data of Piracicaba, SP, Brazil, from 1966 to 2000 were analyzed to establish time distribution models of 60 and 120 min intense rains, during the rainy season from October through March. Time distribution models were assessed by three intervals of twenty minutes duration, for 60 min rains and by four intervals of thirty minutes duration for 120 min rains. The prevailing precipitation model for both, 60 and 120 min rains was a negative exponential distribution, in 85.7 and $50.7 \%$ of cases, respectively. For 60 min rains fractions were distributed as $72.3,21.4$ and $6.2 \%$ of a total $20.7 \mathrm{~mm}$ precipitation. For 120 min rains, the result was $60.1,25.2,11.1$ and $3.6 \%$ of a total $33.3 \mathrm{~mm}$ precipitation, in four successive intervals. Time distribution models for these rains were not affected by the amount of precipitation nor by their duration, in the intervals considered in this study.
\end{abstract}

Key words: intense rains, time distribution, models, probability

\section{INTRODUÇ̃̃O}

A caracterização da variabilidade temporal das chuvas intensas é, ao longo de sua duração, imprescindível para quantificar adequadamente os efeitos ocasionados, pois inúmeros são os problemas de interesse da engenharia, causados por chuvas intensas, de modo especial o controle do escoamento superficial, em áreas urbanas e rurais.

A hidrologia permite a caracterização e a quantificação, de modo preciso, do escoamento superficial originado de chuvas intensas, através de hidrógrafas que correlacionam a variação da descarga com o tempo; para isto, dispõe-se de modelos matemáticos empíricos ou paramétricos até modelos conceituais e determinísticos, que empregam métodos hidrológicos e hidráulicos, além de variáveis consideradas de forma global ou distribuídas espacialmente.

O grande desafio para o engenheiro não é simplesmente quantificar o efeito de um evento hidrológico que acaba de ocorrer mas, essencialmente, a capacidade de prever a ocorrência de eventos extremos e suas conseqüências, da forma mais fiel 
e real possível. Hoje, são cada vez mais utilizados modelos matemáticos que têm sido associados a sistemas de informações geográficas (SIG) que permitem uma descrição detalhada e espacializada das características físicas da área de projeto, porém, nenhum modelo é absolutamente livre de imperfeições, por conta das simplificações e das adaptações necessárias para que possa ser viável e operacional. Um dos grandes inconvenientes em modelos que envolvem a ocorrência de chuvas, é a sua incapacidade de reconhecê-las na sua forma real; assim, todo modelo matemático que ignora ou não distingue a variabilidade da distribuição temporal da chuva, com diferentes durações, apresenta restrições; além disso, para que a previsão hidrológica seja consistente, é desejável se conhecer, "a priori”, o modelo de distribuição de chuva representativo da região climática estudada e de maior probabilidade de ocorrência para o período considerado.

Sentelhas et al. (1998) analisaram e caracterizaram a distribuição horária de chuvas intensas com quatro horas de duração, para Piracicaba, SP, apresentando o modelo de distribuição com maior probabilidade de ocorrência, de outubro a março. Os resultados foram apresentados na forma de histograma, tendo a duração de $4 \mathrm{~h}$, subdividida em quatro intervalos iguais e sucessivos de uma hora. O modelo predominante em $85 \%$ dos casos, entre outubro e março, foi o exponencial negativo, com frações da precipitação total de $69,3 \%$ na primeira, de $16,3 \%$ na segunda, de $9,4 \%$ na terceira e de $5 \%$ na quarta hora, caracterizando uma chuva de distribuição adiantada, frontal ou convectiva.

Em áreas urbanas, chuvas intensas provocam cheias nos sistemas de drenagem naturais e causam picos de escoamento nas galerias pluviais (Righetto, 1998) cujo estudo é fundamental para a determinação da chuva de projeto para esses sistemas. No meio rural, o conhecimento das características das chuvas intensas é de grande importância, uma vez que a sua intensidade é fator expressivo no processo de erosão do solo (Reyes et al., 1993).

Alguns autores, entre eles Wilken (1978) e Greer, citados por Val (1985), apresentaram limites para se considerar uma chuva como intensa. Na prática, a fixação de um valor para se estabelecer chuvas intensas é difícil, uma vez que o impacto pode ser diferente, dependendo do local de incidência, seja em áreas rurais ou urbanas (Pinto, 1999).

Vários trabalhos, entre eles os de Miller et al.(1973), Fedrerick et al. (1977), Vieira e Souza (1983), Goulart et al. (1992) e Mello et al. (1994), vêm sendo realizados com o objetivo de ajustar a uma distribuição teórica os dados de chuva observados, para se determinar os valores de precipitação para os diversos níveis de probabilidade ou, ainda, para se estabelecer a relação intensidade-duração-freqüência da precipitação, porém, na maioria dos casos, os dados de chuva são avaliados como valores pontuais, enquanto deveriam sê-lo na forma de distribuição temporal (Sivapalan \& Blöschl, 1998), para uma efetiva caracterização de sua variabilidade, o que é imprescindível, por exemplo, para a análise de hidrógrafas.

Mello et al. (1994) avaliaram a adequação da distribuição gama, de Erlang, lognormal e de valores extremos às amostras de totais pluviais máximos horários, para Campinas, SP, em que, com os resultados obtidos pelos autores, chegou-se à conclusão de que as distribuições foram adequadas para representar as amostras nos meses analisados, ocorrendo exceção para o mês de janeiro, visto que apenas as distribuições de Erlang e lognormal foram representativas.

Silva et al. (1999) ajustaram modelos teóricos de distribuição de probabilidade aos dados de chuvas intensas nos Estados do Rio de Janeiro e Espírito Santo e estabeleceram a relação intensidade-duração-freqüência da precipitação. Trabalho semelhante foi realizado por Damé et al. (1996) para dados de precipitação anual, máxima e mínima mensal para algumas regiões do Rio Grande do Sul, enquanto em outro trabalho, Robaina (1996) desenvolveu um modelo para se estimar chuvas intensas com durações menores que 24 horas, no Estado do Rio Grande do Sul, que se mostrou adequado quando as chuvas estimadas foram comparadas com as observadas.

À exceção de Sentelhas et al. (1998), raros têm sido os trabalhos no Brasil envolvidos com a caracterização da distribuição temporal de chuvas intensas, ao passo que no exterior este tipo de estudo é bastante comum (Huff, 1967, Preul \& Papadakis, 1973, Pilgrim \& Cordery, 1975, Yen \& Chow, 1980, Olsson \& Berndtsson, 1998 e Burckhardt-Gammeter \& Fankhauser, 1998). Segundo Cruciani (1986) conhecer o modelo de distribuição temporal de chuvas intensas de uma localidade torna mais realista a previsão hidrológica em projetos de engenharia em áreas rurais e urbanas, permitindo a caracterização e a quantificação, com maior precisão, do escoamento superficial, o que pode ser efetuado pela aplicação de métodos de análise de hidrógrafas, como o conhecido método de Sherman e o do Soil Conservation Service, entre outros.

Enfim, é nesse sentido e para se atender a esta necessidade que, essencialmente, residem os objetivos do presente trabalho, com o qual se procura caracterizar o modelo representativo da distribuição temporal de chuvas de duração de 60 e 120 min, em Piracicaba, SP.

\section{MATERIAL E MÉTODOS}

Os dados de precipitação pluvial utilizados no presente estudo foram obtidos no Posto Agrometeorológico da Escola Superior de Agricultura “Luiz de Queiroz”, em Piracicaba, Estado de São Paulo, localizado nas coordenadas geográficas: latitude $22^{\circ} 42^{\prime} \mathrm{S}$, longitude $47^{\circ} 38^{\prime} \mathrm{W}$ e altitude $546 \mathrm{~m}$. O período analisado foi de 1966 a 2000, entre os meses de outubro e março, caracterizando a estação chuvosa, quando ocorre cerca de $78 \%$ do total anual de chuvas.

Os eventos selecionados seguiram os seguintes critérios:

a) para chuvas intensas de $60 \mathrm{~min}$, subdivididas em 3 intervalos de tempo de 20 min cada um:

- Prec $\geq 12 \mathrm{~mm}$.

b) para chuvas intensas de $120 \mathrm{~min}$, subdivididas em 4 intervalos de tempo de 30 min cada um:

- Prec $\geq 15 \mathrm{~mm}$, nos primeiros $60 \mathrm{~min}$, ou

- Prec $\geq 20 \mathrm{~mm}$, durante os $120 \mathrm{~min}$.

Esses critérios foram adotados de acordo com a velocidade de infiltração básica da água nos solos da região de Piracicaba que, segundo Reichardt (1987), é da ordem de $15 \mathrm{~mm} \mathrm{~h}^{-1}$. Deste modo, procurou-se selecionar os eventos com potencial para geração de escoamento superficial. 
Os eventos, subdivididos em intervalos, foram dispostos em histogramas, selecionando-se para análise os casos mais freqüentes de distribuição temporal.

Os dados de precipitação foram analisados considerando-se os valores observados, ordenados de forma crescente, de modo a permitirem a análise de distribuição de freqüência e a busca de uma função de probabilidade teórica que melhor se ajustasse à distribuição amostral. A distribuição de probabilidade utilizada foi a lognormal, com 2 parâmetros, muito utilizada em hidrologia e facilmente transformável em normal (Mello et al., 1994). A função densidade de probabilidade, neste caso, é dada por:

$$
\mathrm{f}(\mathrm{x})=\frac{1}{\sigma \mathrm{x} \sqrt{2 \pi}} \mathrm{e}^{-0,5 \frac{(\ln \mathrm{x}-\mu)^{2}}{\sigma^{2}}} \quad \text { para } 0<\mathrm{x}<\infty
$$

sua função de distribuição é dada por:

$$
F(x)=\int_{0}^{\infty} f(x) d(x)
$$

sendo:

$$
\begin{gathered}
E(x)=e^{\mu+\frac{\sigma^{2}}{2}} \\
\operatorname{Var}(x)=\mu^{2}\left(e^{\sigma^{2}}-1\right)
\end{gathered}
$$

em que:

$\sigma$ - desvio-padrão da distribuição, referente às chuvas intensas em períodos de 60 ou $120 \mathrm{~min}$

$\mu \quad$ - média das chuvas intensas em períodos de 60 ou $120 \mathrm{~min}, \mathrm{~mm}$

$\mathrm{x}$ - chuva intensa diária a ser considerada, $\mathrm{mm}$

$\mathrm{E}(\mathrm{x})$ - esperança matemática

$\operatorname{Var}(\mathrm{x})$ - variância teórica

Utilizou-se, também, a distribuição binomial negativa truncada, dada por:

$$
p(x+1)=f(x)\left(\frac{K+x}{x+1}\right)(1-W)
$$

sendo:

$$
\begin{gathered}
\mathrm{W}=\frac{\overline{\mathrm{X}}}{\mathrm{S}^{2}}\left(1-\mathrm{f} \frac{1}{\mathrm{~N}}\right) \\
\mathrm{K}=\frac{\mathrm{W} \overline{\mathrm{x}}-\mathrm{f} \frac{1}{\mathrm{~N}}}{1-\mathrm{W}}
\end{gathered}
$$

em que:

$\mathrm{x}$ - chuva intensa em período de 60 ou $120 \mathrm{~min}$

$\overline{\mathrm{X}}$ - média dos dados agrupados em classe
$\mathrm{S}^{2} \quad$ - variância amostral

$\mathrm{N}$ - soma das freqüências das chuvas de $1 \mathrm{~h}$

W e K - parâmetros da equação

O ajuste das distribuições teóricas à distribuição amostral, foi avaliado pelo teste qui-quadrado $\left(\chi^{2}\right)$, a nível de significância de 0,05 (Assis et al., 1996).

\section{RESULTADOS E DISCUSSÃO}

A análise preliminar dos dados permitiu identificar-se três tipos predominantes de histogramas de distribuição temporal de chuvas intensas de $60 \mathrm{~min}$, apresentados na Figura 1. O caso 1 , do tipo exponencial negativo, foi o que ocorreu com maior freqüência, em média $85,7 \%$ dos eventos no período analisado, seguido do caso 2 , em 7,2\% dos eventos, e do caso $3, \operatorname{em~} 4,7 \%$.
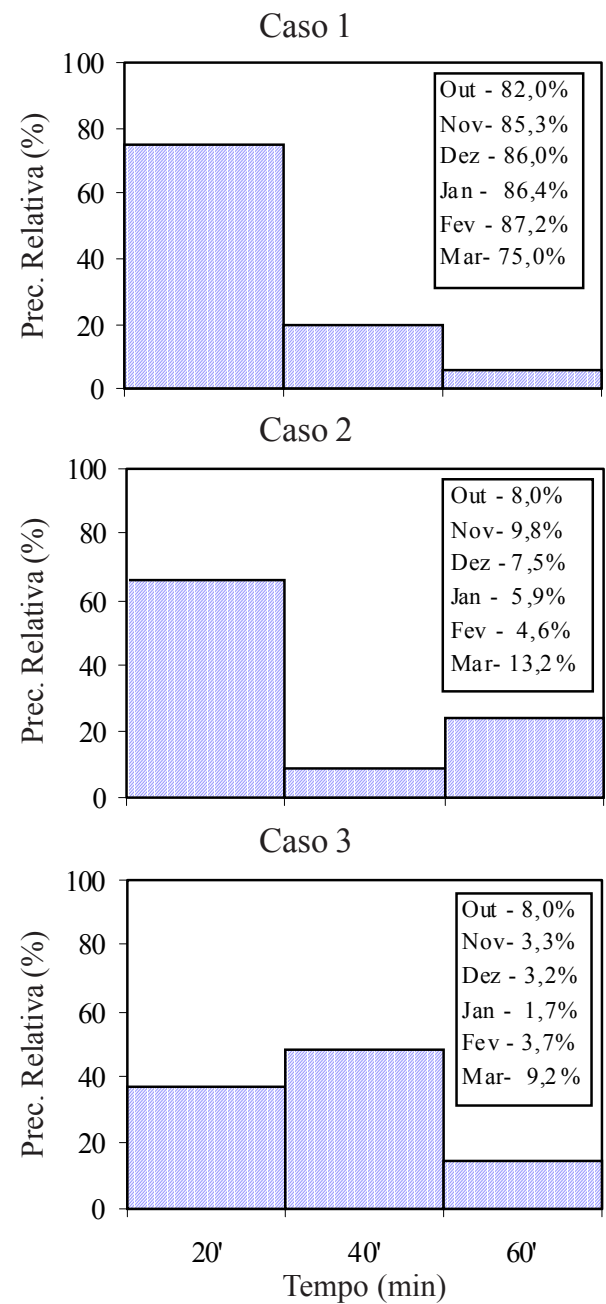

Figura 1. Histogramas de distribuição de chuvas intensas de 60 min, nos meses de outubro a março, em Piracicaba, SP, no período de 1966 a 2000

Na Figura 2 são apresentadas, para cada mês analisado, a precipitação média e sua distribuição em porcentagem. Março foi o mês de maior precipitação média, com 22,9 mm. Esses resultados estão de acordo com aqueles obtidos por Mello et al. (1994) para Campinas, cidade próxima a Piracicaba. Os autores 

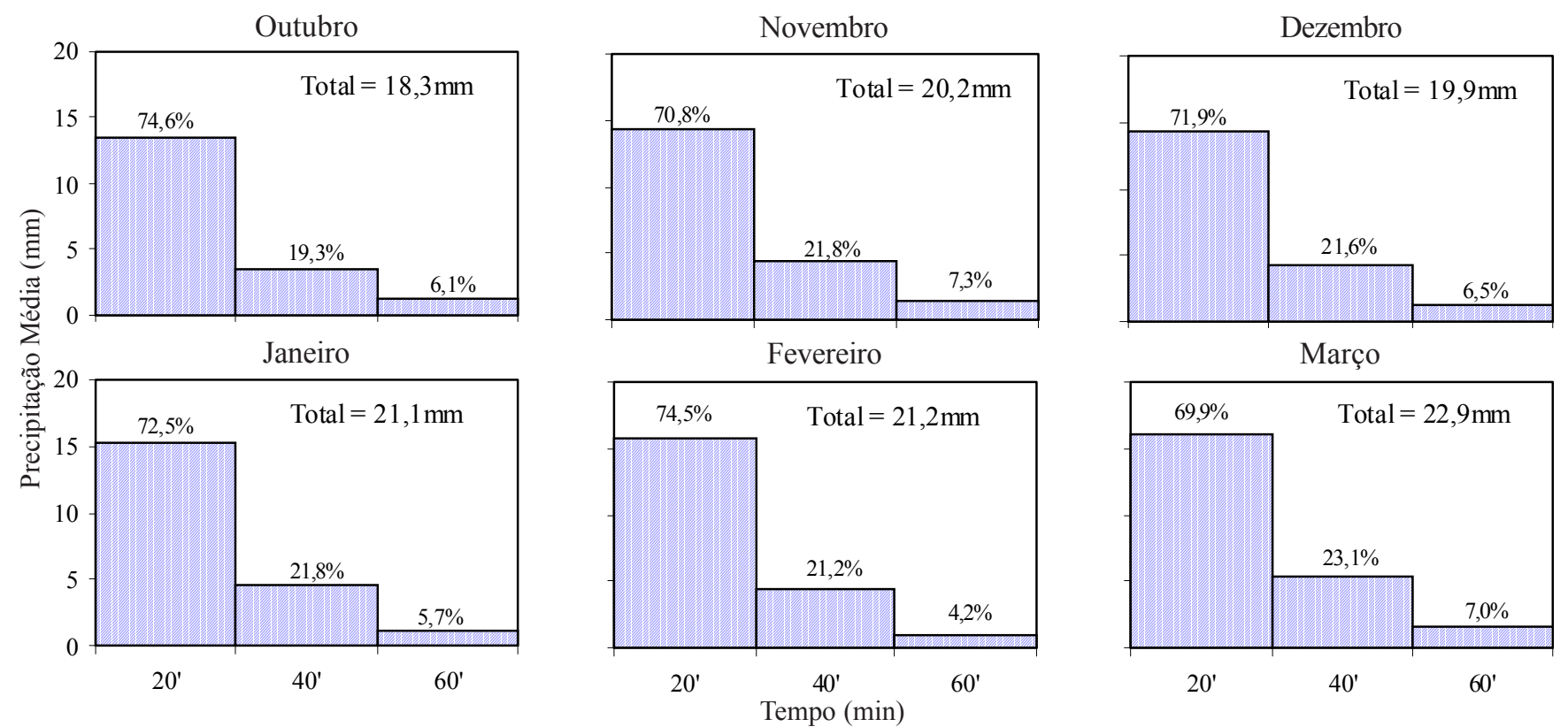

Figura 2. Variação temporal das chuvas intensas de 60 min, nos meses de outubro a março, em Piracicaba, SP, no período de 1966 a 2000

constataram que o mês de março é o mais crítico, em relação à intensidade de chuvas, no período de $60 \mathrm{~min}$.

O modelo geral de distribuição das chuvas intensas de 60 min é apresentado na Figura 3. A chuva média, neste caso, é de $20,7 \mathrm{~mm}$, sendo que $72,3 \%$ desse total ocorrem nos primeiros $20 \mathrm{~min}, 21,4 \%$ nos 20 min seguintes e 6,2\% nos últimos $20 \mathrm{~min}$. Esse padrão é característico das chuvas convectivas, que predominam na região durante o período analisado.

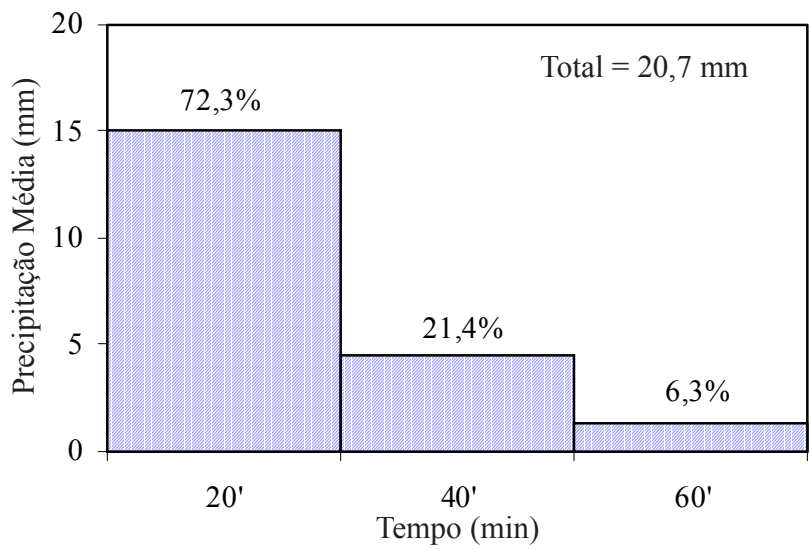

Figura 3. Variação temporal média das chuvas intensas de $60 \mathrm{~min}$, nos meses de outubro a março, em Piracicaba, SP, no período de 1966 a 2000

A distribuição temporal de chuvas de 120 min, subdivididas em 4 intervalos de $30 \mathrm{~min}$, permite 24 arranjos diferentes. No decorrer do período analisado, foram encontrados 15 diferentes arranjos de distribuição temporal de chuvas, entre os quais se selecionaram, para análise, os quatro casos mais freqüentes (Fig. 4). Ao se determinar o modelo de distribuição que representa as chuvas intensas em uma região, deve-se considerar o fato de que uma chuva pode ter uma outra combinação de padrões de duração, intensidade e distribuição. Para as chuvas intensas de $120 \mathrm{~min}$, selecionaram-se quatro casos predomi-

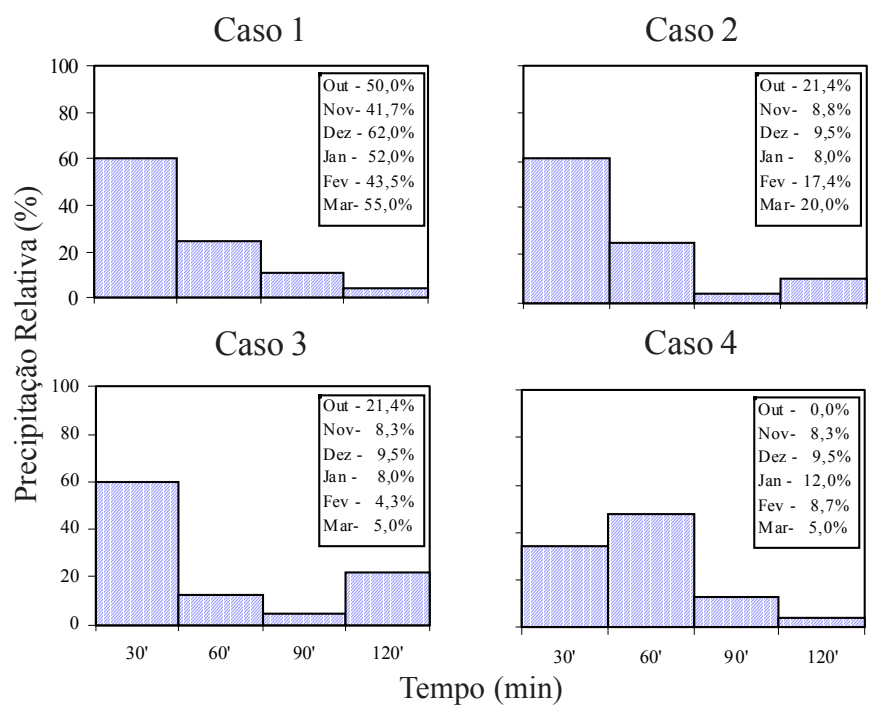

Figura 4. Histogramas predominantes de distribuição de chuvas de $120 \mathrm{~min}$, nos meses de outubro a março, em Piracicaba, SP, no período de 1966 a 2000

nantes de distribuição. Nessa duração, foram encontrados 49 eventos contra 428 eventos de chuvas de $60 \mathrm{~min}$.

Assim, como para as chuvas de $60 \mathrm{~min}$, o tipo mais freqüente (caso 1) das chuvas de 120 min também foi exponencial negativo, com $50,7 \%$ dos eventos no período, seguido do caso 2 , com $14,2 \%$. Os casos 3 e 4 ficaram abaixo de $10 \%$.

$\mathrm{Na}$ Figura 5 tem-se chuvas médias para cada mês e a sua distribuição nos intervalos. Os meses de fevereiro e novembro apresentaram as maiores alturas pluviométricas, com 36,7 e $35,8 \mathrm{~mm}$, respectivamente.

O modelo de distribuição de chuvas de 120 min apresentou média geral de 33,3 mm (Fig. 6), com a seguinte distribuição temporal: $60,1 \%$ no primeiro intervalo de $30 \mathrm{~min}, 25,2 \%$ no segundo, $11,1 \%$ no terceiro e $3,6 \%$ no quarto intervalo. 

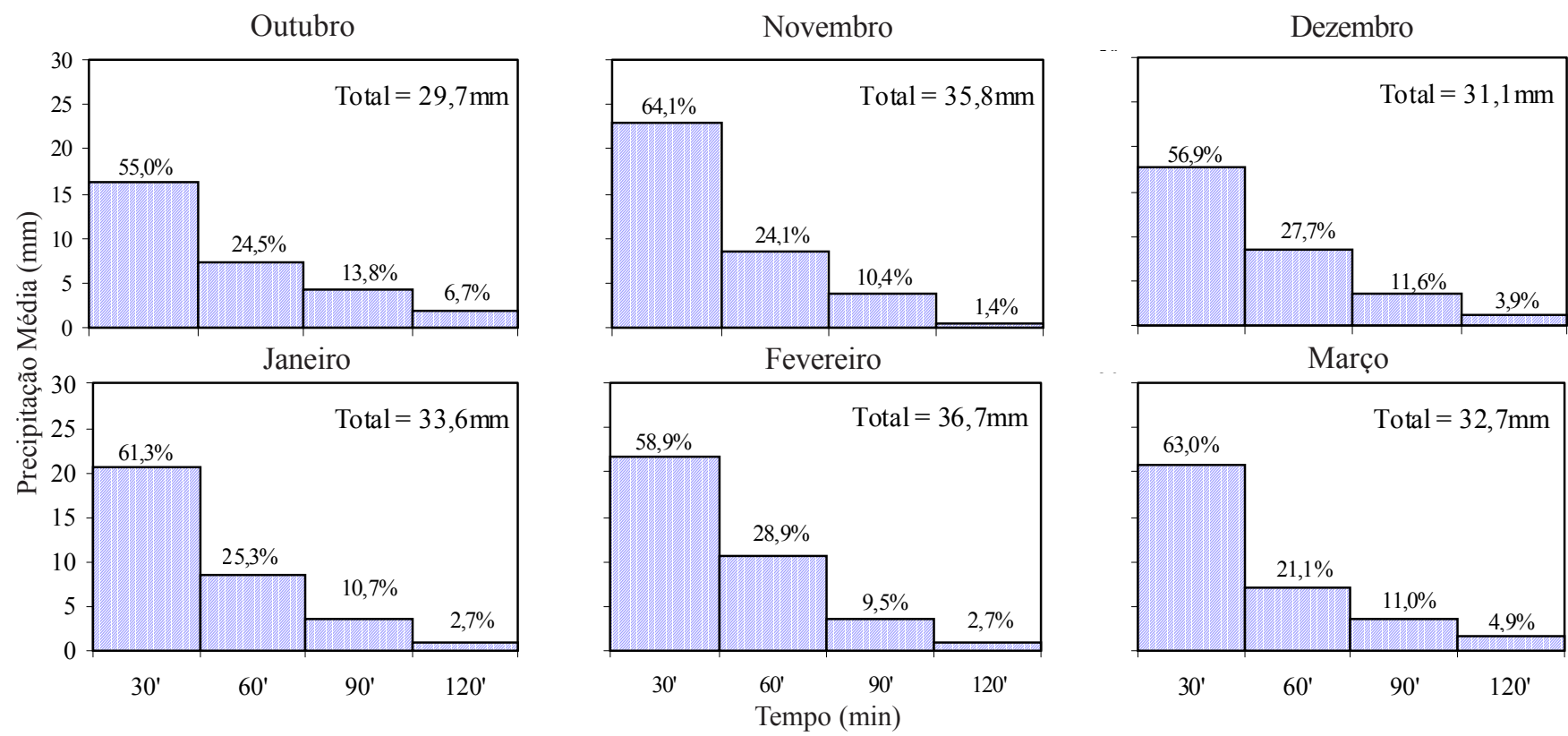

Figura 5. Variação temporal das chuvas intensas de 120 min, nos meses de outubro a março, em Piracicaba, SP, no período de 1966 a 2000

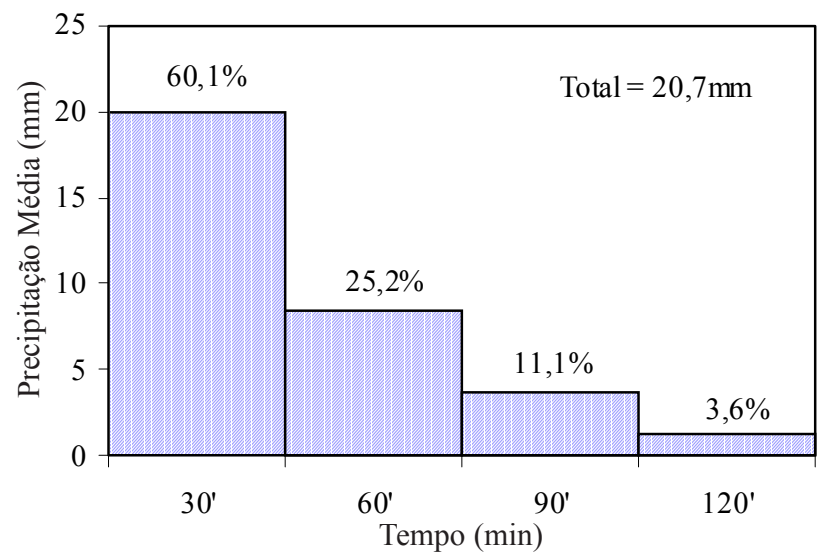

Figura 6. Variação temporal média das chuvas intensas de 120 min, nos meses de outubro a março, em Piracicaba, SP, no período de 1966 a 2000

Sentelhas et al. (1998) analisando chuvas intensas de $4 \mathrm{~h}$ em Piracicaba, SP, nos meses de outubro a março, encontraram o mesmo modelo de distribuição, exponencial negativo, apresentado na Figura 7. Esses resultados mostram que o

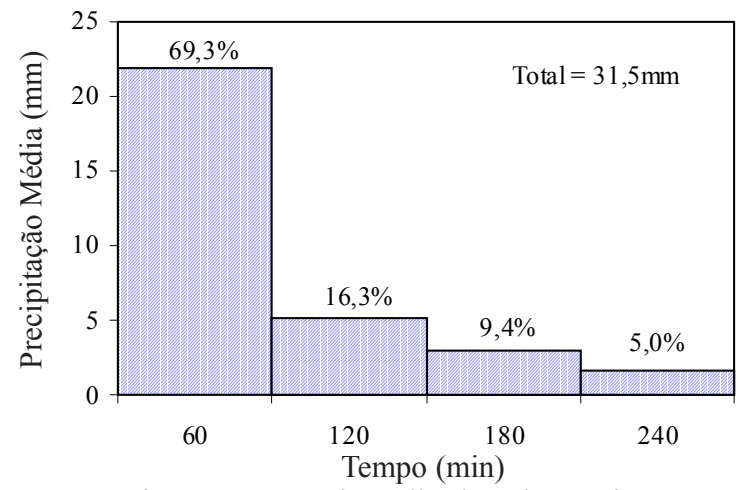

Figura 7. Variação temporal média das chuvas intensas de $4 \mathrm{~h}$ (240 min) nos meses de outubro a março, em Piracicaba, SP, no período de 1966 a 1995 (Sentelhas et al., 1998) modelo de distribuição temporal das chuvas intensas não foi modificado pela altura de chuva nem por sua duração, nos intervalos em questão.

Os dados da Tabela 1 apresentam a estatística descritiva dos eventos de chuva de $60 \mathrm{~min}$. Observa-se haver distribuição com forte assimetria para os eventos de precipitação de $60 \mathrm{~min}$, sendo a média maior que a mediana e a moda e o coeficiente de variação $(\mathrm{CV})$ é alto, entre 28 e $43 \%$.

Tabela 1. Descrição estatística dos eventos de chuvas intensas de $60 \mathrm{~min}$, nos meses de outubro a março, em Piracicaba, SP, no período de 1966 a 2000

\begin{tabular}{lrrrrrrr}
\hline & Out & Nov & Dez & Jan & Fev & Mar & Total \\
\hline N & 41 & 52 & \multicolumn{1}{c}{80} & 102 & 95 & 57 & 428 \\
Média & 18,3 & 20,2 & 19,9 & 21,1 & 21,2 & 22,9 & 20,7 \\
Desvio padrão & 5,2 & 7,5 & 7,6 & 9,05 & 8,8 & 10,6 & 8,6 \\
Máximo & 34,1 & 44,8 & 45,3 & 54,2 & 62,4 & 67,7 & 67,7 \\
Mínimo & 12,0 & 12,4 & 12,0 & 12,0 & 12,0 & 12,2 & 12,0 \\
Moda & 15,1 & 13,6 & 19,1 & 16,0 & 14,1 & 18,0 & 13,8 \\
Mediana & 16,6 & 16,8 & 17,9 & 18,3 & 18,7 & 18,7 & 18,2 \\
CV (\%) & 28,3 & 37,5 & 38,4 & 43,0 & 41,6 & 46,5 & 41,2 \\
\hline N - Número de eventos & & & & & & &
\end{tabular}

Dos modelos de distribuição de freqüência, o que melhor se ajustou à distribuição de freqüência amostral foi a distribuição Binomial Negativa Truncada, na forma de J invertido, com um $\chi^{2}$ calculado de 3,62 , menor que o $\chi^{2}$ tabelado $(11,07)$ sendo adequada a distribuição teórica escolhida. A Figura 8 exibe a distribuição de freqüência empírica e teórica.

As análises estatísticas para as chuvas de 120 min apresentaram um CV total de $29,9 \%$, com valor da média próximo ao da mediana e da moda, mostrando tendência à normalidade (Tabela 2).

A distribuição lognormal foi a que melhor se ajustou à distribuição de freqüência da amostra (Fig. 9). O $\chi^{2}$ calculado, de 2,83 , foi inferior ao tabelado $(7,82)$. 


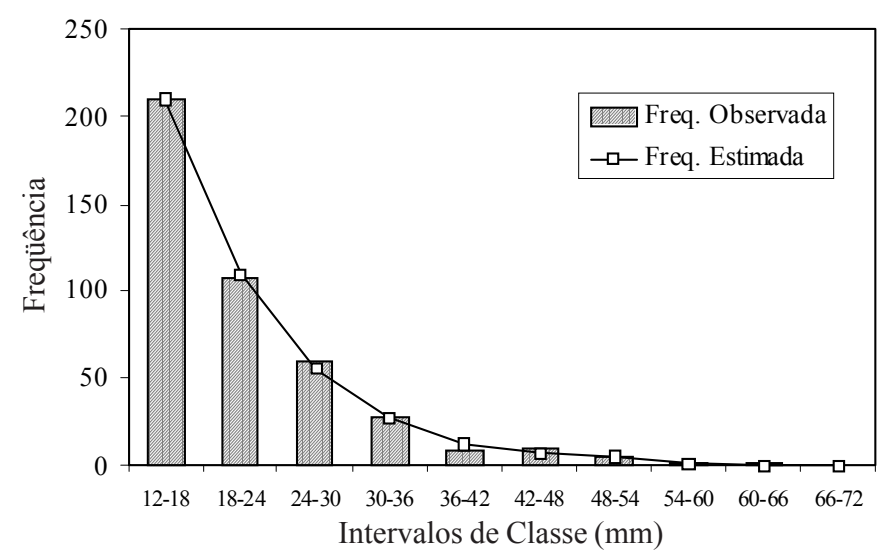

Figura 8. Freqüência observada e estimada pela função de distribuição de probabilidade Binomial Negativa Truncada, para dados de chuvas com duração de 60 min, para Piracicaba, SP, de 1966 a 2000

Tabela 2. Descrição estatística dos eventos de chuvas intensas (N) de 120 min, nos meses de outubro a março, em Piracicaba, SP, no período de 1966 a 2000

\begin{tabular}{lccccccc}
\hline & Out & Nov & Dez & Jan & Fev & Mar & Total \\
\hline $\mathrm{N}$ & 7 & 5 & 13 & 13 & 10 & 11 & 59 \\
Média & 29,7 & 35,8 & 31,2 & 33,6 & 36,7 & 32,7 & 33,2 \\
Desvio padrão & 5,9 & 7,0 & 10,7 & 10,9 & 13,1 & 8,2 & 9,9 \\
Máximo & 37,8 & 43,0 & 51,5 & 50,2 & 66,6 & 47,3 & 66,6 \\
Mínimo & 20,5 & 26,2 & 15,0 & 18,8 & 22,3 & 21,8 & 15,0 \\
Moda & - & - & - & - & - & - & 33,1 \\
Mediana & 29,1 & 34,9 & 28,1 & 33,1 & 36,3 & 32,9 & 32,9 \\
Cv (\%) & 19,7 & 19,6 & 34,3 & 32,4 & 35,7 & 25,1 & 29,9 \\
\hline N - Número de eventos & & & & & & &
\end{tabular}

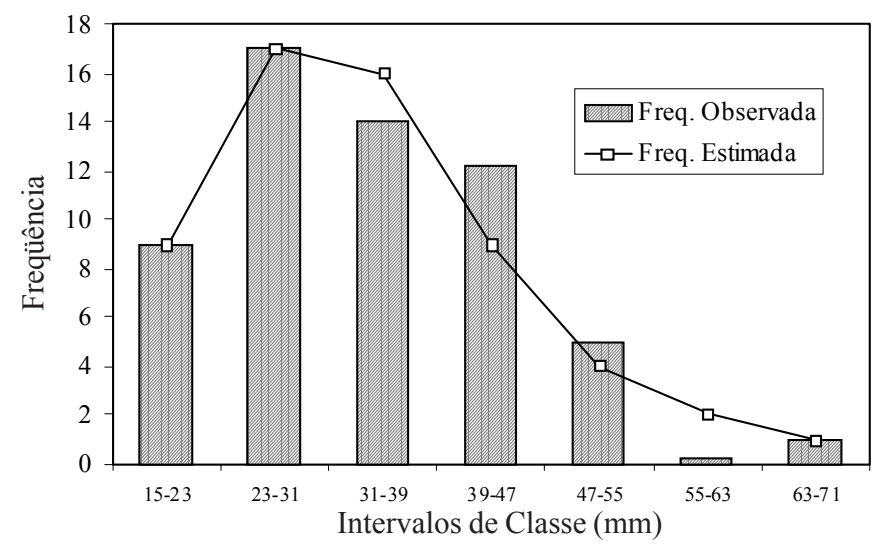

Figura 9. Freqüência observada e estimada pela função de distribuição de probabilidade Lognormal, para dados de chuvas intensas, em período de $120 \mathrm{~min}$, para Piracicaba, SP, de 1966 a 2000

Na Tabela 3 são apresentadas, para chuvas de 60 e 120 min, com intervalos de classe de $6 \mathrm{~mm}$, as probabilidades empírica e teórica de ocorrência de um evento de chuva igual ou maior a um valor, de acordo com uma distribuição Binomial Negativa Truncada e Lognormal, respectivamente.

Observa-se, tanto para chuvas intensas de 60 min quanto para as de $120 \mathrm{~min}$, decréscimo da probabilidade de ocorrência com a intensidade de precipitação, resultados também obtidos por Sentelhas et al. (1998) e Mello et al. (1994).
Tabela 3. Probabilidade (\%) empírica e teórica de ocorrência de valores de chuvas máximas maiores ou iguais a dado valor, utilizando-se a distribuição Binomial Negativa Truncada, para chuvas de 60 min, e a distribuição Lognormal, para chuvas de $120 \mathrm{~min}$

\begin{tabular}{ccccccc}
\hline \multicolumn{2}{c}{ Chuvas } & Intensas de 60 min & & \multicolumn{3}{c}{ Chuvas Intensas de 120 min } \\
\cline { 1 - 2 } $\begin{array}{c}\text { Chuva } \\
(\mathrm{mm})\end{array}$ & $\begin{array}{c}\mathrm{P}(\mathrm{x}) \\
\text { Empírica }\end{array}$ & $\begin{array}{c}\mathrm{P}(\mathrm{x}) \\
\text { Teórica }\end{array}$ & & $\begin{array}{c}\text { Chuva } \\
(\mathrm{mm})\end{array}$ & $\begin{array}{c}\mathrm{P}(\mathrm{x}) \\
\text { Empírica }\end{array}$ & $\begin{array}{c}\mathrm{P}(\mathrm{x}) \\
\text { Teórica }\end{array}$ \\
\hline $12-18$ & 49,3 & 49,3 & & $15-23$ & 15,3 & 15,3 \\
$18-24$ & 24,8 & 25,5 & & $23-31$ & 30,5 & 30,5 \\
$24-30$ & 13,8 & 12,9 & & $31-39$ & 23,7 & 27,1 \\
$30-36$ & 6,5 & 6,3 & & $39-47$ & 20,3 & 15,3 \\
$36-42$ & 1,9 & 3,0 & & $47-55$ & 8,5 & 6,8 \\
$42-48$ & 2,3 & 1,6 & & $55-63$ & 0,0 & 3,4 \\
$48-54$ & 0,7 & 0,7 & & $63-71$ & 1,7 & 1,7 \\
$54-60$ & 0,2 & 0,5 & & & & \\
$60-66$ & 0,2 & 0,2 & & & & \\
$66-72$ & 0,2 & 0,0 & & & & \\
\hline
\end{tabular}

\section{CONCLUSÕES}

Os resultados obtidos permitem as seguintes conclusões:

1. Os histogramas de chuvas intensas de 60 e de $120 \mathrm{~min}$ de duração, representativos dos meses chuvosos (de outubro a março), apresentaram distribuição temporal do tipo exponencial negativa, em 85,7 e $50,7 \%$ dos casos, respectivamente.

2. Para as chuvas de $60 \mathrm{~min}, 72,3 \%$ do total precipitado ocorrem nos primeiros $20 \mathrm{~min}, 21,4 \%$ nos $20 \mathrm{~min}$ seguintes e o restante $(6,2 \%)$ ao longo do último intervalo de $20 \mathrm{~min}$. Para as chuvas de 120 min, a distribuição ao longo do tempo é de $60,1 \%$ no primeiro, $25,2 \%$ no segundo, $11,1 \%$ no terceiro e $3,6 \%$ no quarto, intervalo de $30 \mathrm{~min}$.

3. O modelo de distribuição temporal das chuvas intensas de 60 min e de 120 min de duração é muito semelhante ao modelo de distribuição de chuvas intensas de 4 h (240 min) indicando, que para a cidade de Piracicaba, prevalecem as chuvas de distribuição adiantada, independentemente do total e da duração da precipitação.

\section{LITERATURA CITADA}

Assis, F.N.; Arruda, H.V.; Pereira, A.R. Aplicações de estatística à climatologia. Teoria e Prática. Pelotas: Ed. Universitária/ UFPel. 1996. 161p.

Burckhardt-Gammeter, S.; Fankhauser, R. Analysis of rainfall time series with regard to temporal disaggregation for use in urban hydrology. Water Science and Technology, London, v.37, n.11, p.65-72, 1998.

Cruciani, D.E. A drenagem na agricultura. São Paulo: Ed. Nobel, 1986. 337p.

Damé, R.C.F.; Teixeira, C.F.A.; Souto, M.V. Análise de freqüência dos dados de precipitação pluvial de algumas estações agroclimatológicas da região Sul do Rio Grande do Sul. Ciência Rural, Santa Maria, v.26, n.3, p.351-355, 1996.

Frederick, R.H.; Myers, V.A.; Auciello, E.P. Five to 60-minute precipitation frequency for eastern and central United States. NOAA Silver Spring, MA: National Weather Service, 1977. Technical Memo NWS HYDRO-35 
Goulart, J.P.; Maestrini, A.P.; Neibel, A.L. Relação intensidadeduração-freqüência de chuvas em Pelotas, RS. Revista Brasileira de Meteorologia, São Paulo, v.7, n.1, p.543-552, 1992.

Huff, F.A. Time distribution of rainfall in heavy storms. Water Resource Research, Washington, v.3, n.4, p.1007-1019, 1967.

Mello, M.H.A.; Arruda, H.V.; Ortolani, A.A. Probabilidade de ocorrência de totais pluviais máximos horários, em Campinas, São Paulo. Revista IG, São Paulo, v.15, n.1/2, p. 59-67, 1994.

Miller, J.F.; Frederick, R.H.; Tracey, R.J. Precipitation-frequency Atlas of the conterminous western United States. NOAA Atlas 2, Silver Spring, MA: National Weather Service, 1973.

Olsson, J.; Berndtsson, R. Temporal rainfall disaggregation based on scaling properties. Water Science and Technology, London, v.37, n.11, p.73-79, 1998.

Pilgrim, D.H.; Cordey, I. Rainfall temporal patterns for design floods. Journal of the Hydraulics Division, Reston, v.101, n.HY1, p.81-95, 1975.

Pinto, F.R.L. Equações de intensidade-duração-freqüência da precipitação para os Estados do Rio de Janeiro e Espírito Santo: Estimativa e especialização. Viçosa, MG: UFV, 1999. 70p. Dissertação Mestrado

Preul, H.D.;Papadakis, C.N. Development of design storm hyetographs for Cincinnati, Ohio. Water Resource Bulletin, Middleburg, v.9, n.2, p.291-300, 1973.

Reichardt, K. A água em sistemas agrícolas. São Paulo: Editora Mande, 1987. 188p.

Reyes, M.R.; Bengtson, R.L.; Robbins, K.D. Rip and Wmrip. New measures of rainfall intensity distribution. Transactions of the ASAE, St Joseph, v.36, n.6, p.1619-1623, 1993.
Righetto, A.M. Hidrologia e recursos hídricos. São Carlos: EESC/USP, 1998. 819p.

Robaina, A.D. Modelo para geração de chuvas intensas no Rio Grande do Sul. Revista Brasileira de Agrometeorologia, Santa Maria, v.4, n.2, p.95-98, 1996.

Sentelhas, P.C.; Cruciani, D.E.; Pereira, A.S.; Villa Nova, N.A. Distribuição horária de chuvas intensas de curta duração: um subsídio ao dimensionamento de projetos de drenagem superficial. Revista Brasileira de Meteorologia, Jaboticabal, v.13, n.1, p.45-52, 1998.

Silva, D.D.; Pinto, F.R.L.; Prusk, F.F., Pinto, F.A. Estimativa e espacialização dos parâmetros da equação de intensidadeduração-freqüência da precipitação para o Rio de Janeiro e o Espírito Santo. Engenharia Agrícola, Jaboticabal, v.18, n.3, p.11-21, 1999.

Sivapalan, M.; Blöschl, G. Tranformation of point rainfall to areal rainfall: intensity-duration-frequency curves. Journal of Hydrology, Amsterdam, v.204, n.1-4, p.150-167, 1998.

Val, L.A. Avaliação dos índices de erosividade das chuvas no município de Lavras, MG. Lavras, MG: ESAL, 1985. 72p. Dissertação Mestrado

Vieira, D.B.; Souza, C.Z. Análise das relações intensidadeduração-freqüência das chuvas intensas para Ribeirão Preto. Item, Campinas, v.14, n.9, p.20-29, 1983.

Wilken, P.S. Engenharia de drenagem superficial. São Paulo: Companhia de Tecnologia de Saneamento Ambiental, 1978. $487 \mathrm{p}$.

Yen, B.C.; Chow, V.T. Design hyetographs for small drainage strucutures. Journal of the Hydraulics Division, Reston, v.106, n.HY6, p.1055-1076, 1980. 